\title{
Xantomas Eruptivos: Manifestação Cardinal de Distúrbio Metabólico Grave
}

\section{Eruptive Xanthomas: A Cardinal Manifestation of Serious Metabolic Disease}

\author{
Luís SANTIAGO ${ }^{1}$, André PINHO ${ }^{1}$, José Carlos CARDOSO ${ }^{1}$ \\ Acta Med Port 2018 Apr;31(4):219-222 - https://doi.org/10.20344/amp.9126
}

\begin{abstract}
RESUMO
Os xantomas eruptivos resultam de uma deposição rápida intracelular e dérmica de lípidos e constituem um importante sinal de risco cardiovascular, com impacto prognóstico. Os autores descrevem o caso de um homem de 47 anos com múltiplas pápulas distribuídas nas nádegas, região lombar, parede abdominal, cotovelos e pescoço com dois meses de evolução, cupuliformes, amareladas, firmes e pouco pruriginosas. Os antecedentes pessoais incluíam abuso etílico, diabetes mellitus tipo 2 e dislipidemia não medicadas, além de início recente de terapêutica com olanzapina por síndrome depressivo. Os exames complementares mostraram hipertrigliceridemia (7474 mg/dL), hipercolesterolemia (1054 mg/dL) e hemoglobina A1c de 12,1\%. O estudo histopatológico de uma pápula do flanco esquerdo revelou numerosos histiócitos xantomizados na derme confirmando o diagnóstico de xantomas eruptivos. No nosso caso, a introdução da olanzapina poderá ter agravado os antecedentes de doença metabólica, contribuindo assim para o rápido aparecimento das lesões cutâneas. O reconhecimento atempado dos xantomas eruptivos pode ter um profundo impacto no diagnóstico, tratamento e prognóstico, por conduzir à pesquisa de causas secundárias.
\end{abstract}

Palavras-chave: Doenças da Pele; Hipertrigliceridemia; Olanzapina; Xantomatose

\section{ABSTRACT}

Eruptive cutaneous xanthomas develop as a result of rapid intracellular and dermal deposition of lipids and are a cardinal sign of high cardiovascular risk. We describe the case of a 47-year-old male with multiple dome-shaped, yellowish and firm, slightly pruriginous papules distributed over the buttocks, lumbar region, abdominal wall, elbows and neck, for about two months. Previous medical history included alcohol abuse, non-medicated type 2 diabetes mellitus, dyslipidemia and recent onset of therapy with olanzapine for depressive syndrome. Work-up revealed hypertriglyceridemia (7474 mg/dL), hypercholesterolemia (1054 mg/dL) and hemoglobin A1c of $12.1 \%$. Histopathologic examination of an abdominal papule showed clusters of foamy histiocytes in the dermis confirming the diagnosis of eruptive xanthomas. In this case, the introduction of olanzapine may have aggravated the previous history of metabolic disease which may have led to the rapid onset of skin lesions. Proper recognition of eruptive xanthomas can have a profound impact on diagnosis, treatment and prognosis of patients who suffer from an underlying systemic disease.

Keywords: Hypertriglyceridemia; Olanzapine; Skin Diseases; Xanthomatosis

\section{INTRODUÇÃO}

Os xantomas cutâneos (XC) são lesões raras que resultam de uma deposição rápida de lípidos a nível dérmico com inflamação cutânea secundária. ${ }^{1}$ Manifestam-se, habitualmente, na idade adulta, com exceção dos casos associados à hipercolesterolemia familiar. ${ }^{2}$

OS XC apresentam morfologias e etiopatogenias distintas. Podem manifestar-se clinicamente como xantomas eruptivos (XE), xantomas tuberosos, xantomas tendinosos, xantomas verruciformes, xantomas planos ou xantelasmas. A maioria associa-se a alterações primárias ou secundárias do metabolismo das lipoproteínas, com exceção de alguns xantomas verruciformes e planos. Em alguns casos, o subtipo de xantoma é preditivo de um distúrbio lipídico específico, constituindo um importante sinal de risco cardiovascular, com impacto prognóstico. ${ }^{1-3}$

Os XE ocorrem habitualmente no contexto de hiperquilomicronemia e hipertrigliceridemia causadas por um defeito primário, secundário ou ambos. Os níveis de triglicerídeos habitualmente excedem $3000-4000 \mathrm{mg} / \mathrm{dL}^{2}{ }^{2}$ As formas primárias correspondem à hiperquilomicronemia familiar, à hipertrigliceridemia familiar endógena e à hiperlipidemia primária mista. As formas secundárias encontram- -se associadas à obesidade, alcoolismo crónico, diabetes mellitus não controlada e algumas medicações sistémicas. Raramente, os XE podem estar associados a insuficiência renal grave, hipotiroidismo, neoplasias hematológicas e doenças autoimunes colestáticas (Tabela 1). $\cdot^{1-5}$

Apesar da elevada prevalência da dislipidemia na população geral, apenas uma minoria desenvolve XC, estimando-se que ocorra em $0,001 \%-0,01 \%$ da população. ${ }^{2,6}$ Os motivos pelos quais isto se verifica, assim como o seu mecanismo de formação, ainda são desconhecidos. Nos xantomas relacionados com dislipidemia, assume-se que a elevação dos níveis séricos de lipoproteínas resulta na sua extravasão através dos capilares dérmicos, seguida de fagocitose por macrófagos que levam ao aparecimento das células xantomizadas (foam cells) caracteristicamente observadas na histologia destas lesões. ${ }^{2,3}$

\section{CASO CLÍNICO}

Homem de 47 anos é referenciado à Dermatologia por erupção papular disseminada e simétrica com dois meses de evolução. Ao exame objetivo apresentava múltiplas pápulas distribuídas pelas nádegas, região lombar,

\footnotetext{
1. Serviço de Dermatologia. Centro Hospitalar e Universitário de Coimbra. Coimbra. Portugal.

$\triangle$ Autor correspondente: Luís Santiago. luisgsantiago2@gmail.com

Recebido: 25 de abril de 2017 - Aceite: 07 de dezembro de 2017 | Copyright @ Ordem dos Médicos 2018
} 
Tabela 1 - Causas de xantomas eruptivos

\begin{tabular}{l}
\hline Causas Primárias \\
\hline Deficiência primária de lipoproteína lipase ou apo C-II \\
Hipertrigliceridemia familiar endógena \\
Hiperlipidemia primária mista \\
\hline Causas Secundárias \\
\hline
\end{tabular}

Diabetes mellitus tipo 2

Alcoolismo

Obesidade

Fármacos (antipsicóticos de $2^{\mathrm{a}}$ geração, glucocorticóides, terapêutica hormonal de substituição, contracetivos orais, tamoxifeno, ciclosporina, retinóides, antiretrovirais e altas doses de diuréticos tiazídicos e de antagonistas beta-adrenérgicos)

Hipotiroidismo

Insuficiência renal grave

Doenças autoimunes colestáticas (colangite esclerosante primária ou a colangite biliar primária)

Neoplasias hematológicas (mieloma múltiplo)

abdómen inferior e superfície extensora dos membros superiores e pescoço, cupuliformes, amareladas, com 2 - 4 $\mathrm{mm}$, firmes e algumas das quais rodeadas por um halo inflamatório, (Fig.s 1 A - D).

$\mathrm{O}$ doente tinha como antecedentes pessoais obesidade, abuso etílico, além de diabetes mellitus tipo 2 e dislipidemia diagnosticadas há cinco anos, mas não controladas, por falta de adesão à terapêutica. Concomitantemente, tinha iniciado três meses antes olanzapina, por depressão reativa com comportamento heteroagressivo. Negava história familiar de dislipidemia ou história pregressa de
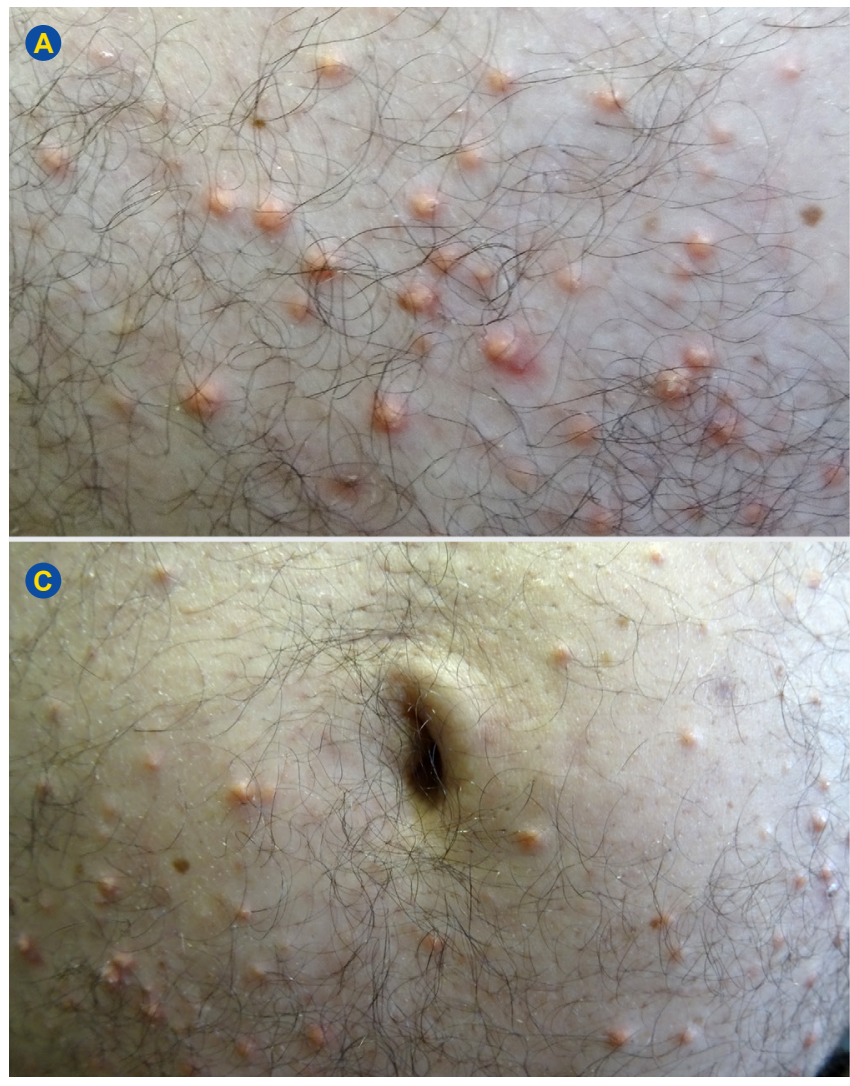

Figura 1 - Xantomas eruptivos: pormenor das lesões cutâneas (A), região lombar (B), abdómen (C) e cotovelo (D) complicações cardiovasculares.

A punção venosa para estudo laboratorial mostrou sangue de aspeto quiloso e analiticamente apresentava hipertrigliceridemia de $7474 \mathrm{mg} / \mathrm{dL}$, hipercolesterolemia de 1054 $\mathrm{mg} / \mathrm{dL}$ e hemoglobina A1c (HbA1c) de 12,1\%. Não apresentava alterações da função renal ou hepática. A avaliação dos anticorpos anti-ilhéus de Langerhans, anti-insulina, anti-IA2, anti GAD65 foi negativa e o doseamento das hormonas tiroideias foi normal.

As alterações encontradas eram de magnitude superior àquelas encontradas em análises de rotina sete meses
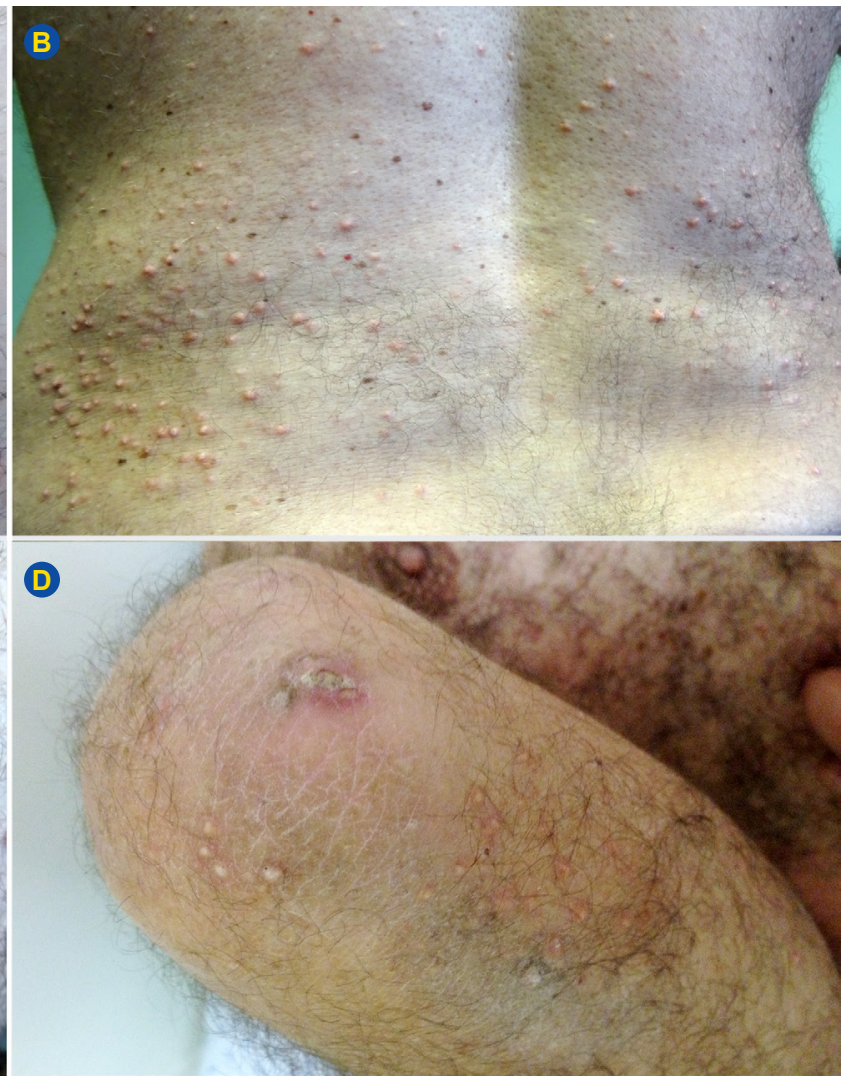


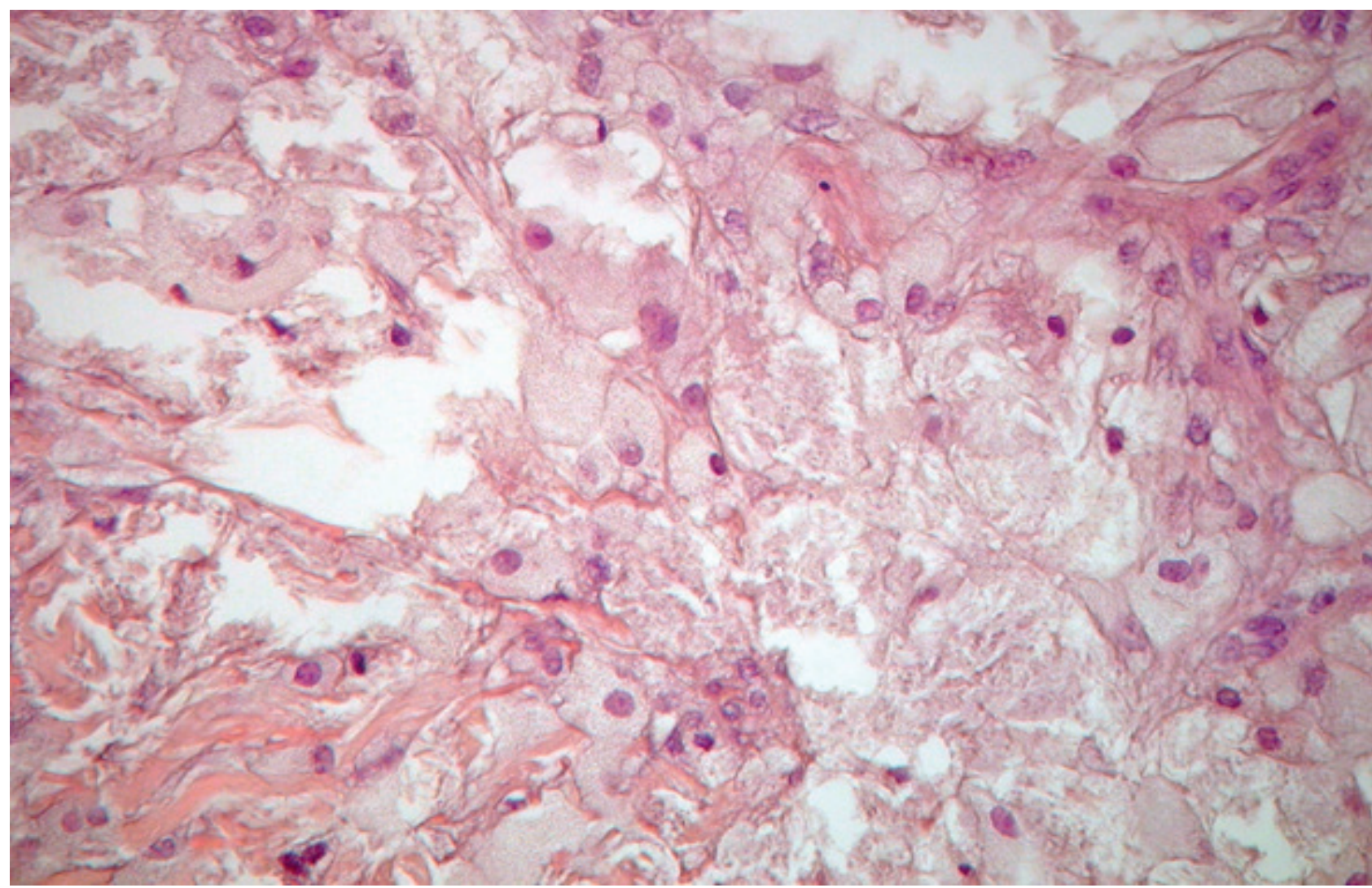

Figura 2 - Histologia das lesões com numerosos histiócitos xantomizados com citoplasma abundante, acompanhados de infiltrado inflamatório linfomononucleado circundante

antes, em que apresentava hipertrigliceridemia (998 mg/ $\mathrm{dL}$ ), hipercolesterolemia (267 mg/dL) e HbA1c de 9,8\%, mas sem lesões cutâneas sugestivas de xantomas.

A avaliação histopatológica de uma pápula do flanco esquerdo revelou infiltração da derme superficial e média por numerosos histiócitos xantomizados rodeados por infiltrado inflamatório linfomononucleado (Fig. 2).

Confirmou-se, portanto, o diagnóstico de XE associado a causas secundárias de dislipoproteinemias. Apesar de não ser possível excluir uma causa primária subjacente, resultando na maioria dos casos em padrão de hiperlipidemia tipo IV ou V, esta torna-se pouco provável, dada a ausência de história familiar, a raridade da hiperquilomicronemia familiar e os valores de triglicerídeos superiores a $1000 \mathrm{mg} /$ $\mathrm{dL}$, habitualmente não observados na hipertrigliceridemia familiar endógena.

O doente iniciou dieta hipolipídica, hipoglicémica e cessação etílica, assim como terapêutica com rosuvastatina, fenofibrato, ácido gordo omega-3 e insulinoterapia. Após avaliação por psiquiatria suspendeu a olanzapina. Os valores de triglicerídeos, colesterol total e $\mathrm{HbA} 1 \mathrm{c}$ diminuíram rapidamente durante os primeiros 14 dias de terapêutica, com normalização após cinco meses (148 mg/dL, 169 mg/ $\mathrm{dL}$ e $6,2 \%$, respetivamente). Verificou-se total regressão das lesões cutâneas 30 dias após início da terapêutica dirigida (Fig.s 3A, 3B).

\section{DISCUSSÃO}

O diagnóstico de XE é essencialmente clínico, apoiando-se na comprovação histológica e na presença de hipertrigliceridemia. As lesões tipicamente apresentam-se como pápulas amareladas localizadas sobretudo na região nadegueira e superfície extensora dos membros. ${ }^{2}$

Perante a possibilidade diagnóstica de XE é mandatória a avaliação dos valores de triglicerídeos e aferir causas primárias e secundárias de dislipidemia. $\mathrm{O}$ tratamento dos XE envolve a correção das causas subjacentes de dislipidemia. Com a redução dos triglicerídeos para níveis aceitáveis, verifica-se regressão das lesões eruptivas em semanas, sem formação de cicatrizes. Não é incomum a recidiva das lesões dermatológicas se ocorrer deterioração metabólica. ${ }^{3,6}$

No caso descrito, existia uma associação de várias causas secundárias (obesidade, consumo etílico, diabetes mellitus tipo 2) responsáveis pela presença de uma dislipidemia mista, já presente em controlo analítico anterior ao aparecimento dos XE. Nestas patologias a hipertrigliceridemia ocorre por dois mecanismos: por um lado, a insulinorresistência culmina numa deficiência adquirida da lipoproteína lipase; por outro, há um aumento da produção hepática de VLDL. ${ }^{4}$

O precipitante final para a elevação acentuada dos valores de triglicerídeos poderá ser explicado, provavelmente, pelo início da terapêutica com olanzapina, com as suas interações a nível da ação da insulina. Este fármaco atua 

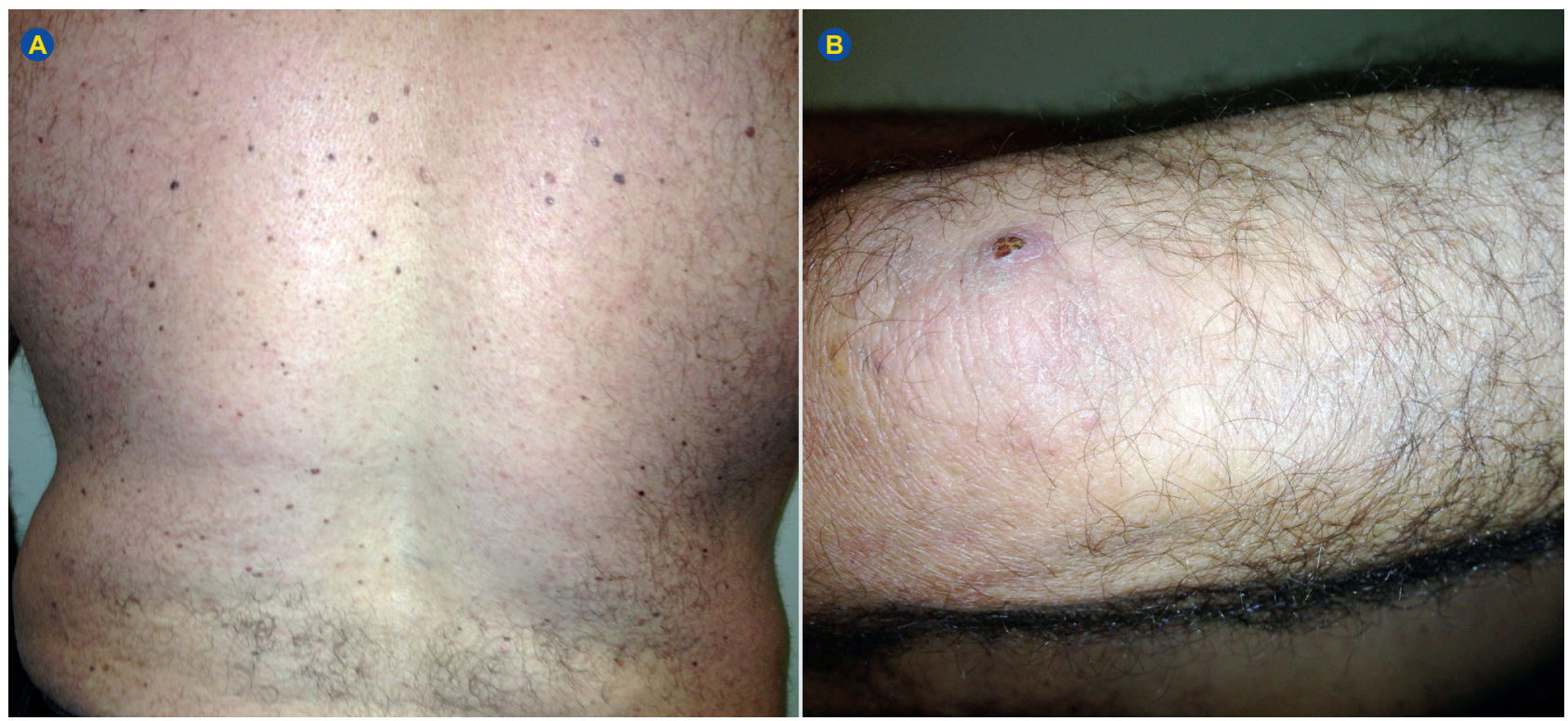

Figura 3 - Regressão das lesões cutâneas a nível lombar (A) e cotovelo (B), dois meses após início do tratamento

através da inibição da recaptação de serotinina que tem um duplo papel no metabolismo da glicose: a nível central, reduzindo a resposta à hiperglicemia, e perifericamente nas células pancreáticas, diminuindo a secreção de insulina. ${ }^{7-9}$ Este antagonismo provocado pela olanzapina resulta numa insuficiência relativa de insulina, com interferência no metabolismo da glicose e das lipoproteínas, levando à formação de XE. ${ }^{9,10}$ Este mecanismo parece necessitar de um defeito pré-existente na secreção de insulina, ${ }^{7}$ tal como no nosso caso clínico.

Apesar da dislipidemia associada a antipsicóticos de segunda geração ser elevada (superior a 30\%), este efeito secundário é raramente relatado na literatura, não sendo possível prever quais os indivíduos que irão apresentar os efeitos secundários metabólicos mais severos. Os casos são clinicamente exuberantes (com valores de triglicerídeos superiores a $3000 \mathrm{mg} / \mathrm{dL}$ ), contudo não é estritamente necessária a retirada do fármaco para que se verifique melhoria clínico-laboratorial. .,9 $^{2}$

As manifestações cutâneas podem ser um sinal de alarme, facilmente identificável, de doenças sistémicas, sendo os XC um desses exemplos. No caso clínico descrito, a introdução de olanzapina poderá ter agravado as doenças metabólicas prévias, resultando no aparecimento dos XE, o que evidencia a necessidade de excluir causas ambientais subjacentes. O estabelecimento do diagnóstico e o

\section{REFERÊNCIAS}

1. Parker F. Xanthomas and hyperlipidemias. J Am Acad Dermatol. 1985;13:1-30.

2. Bolognia JL, Jorizzo JL, Schaffer JV. Dermatology. $3^{\text {rd }}$ ed. London: Elsevier; 2012.

3. Russo GG. Hyperlipidemias. Clin Dermatol. 1996;14:367-74.

4. Loeckermann S, Braun-Falco M. Eruptive xanthomas in association with metabolic syndrome. Clin Exp Dermatol. 2010;35:565-6.

5. Garg A, Simha V. Update on dyslipidemia. J Clin Endocrinol Metab. 2007;92:1581-9.

6. Andrews S, Byrom L, Adikari C, Strungs I, Muir J. Eruptive xanthomas: a collaborative approach using tele-dermatology. Aust Fam Physician. estudo da doença metabólica subjacente permitiram iniciar tratamento médico célere de forma a prevenir complicações relacionadas com a hipertrigliceridemia grave, nomeadamente pancreatite aguda e doenças cardiovasculares.

\section{PROTECÇÃO DE PESSOAS E ANIMAIS}

Os autores declaram que os procedimentos seguidos estavam de acordo com os regulamentos estabelecidos pelos responsáveis da Comissão de Investigação Clínica e Ética e de acordo com a Declaração de Helsínquia da Associação Médica Mundial.

\section{CONFIDENCIALIDADE DOS DADOS}

Os autores declaram ter seguido os protocolos do seu centro de trabalho acerca da publicação de dados.

\section{CONSENTIMENTO DO DOENTE}

Obtido.

\section{CONFLITOS DE INTERESSE}

Os autores declaram não terem qualquer conflito de interesse relativamente ao presente artigo.

\section{FONTES DE FINANCIAMENTO}

Os autores declaram não ter recebido subsídios ou bolsas para a elaboração do artigo.

2016;45:57-8

7. Avram AM, Patel V, Taylor HC, Kirwan JP, Kalhan S. Euglycemic clamp study in clozapine-induced diabetic ketoacidosis. Ann Pharmacother. 2001;35:1381-7.

8. Meyer JM, Koro CE. The effects of antipsychotic therapy on serum lipids: a comprehensive review. Schizophr Res. 2004;70:1-17.

9. Chang HY, Ridky TW, Kimball AB, Hughes E, Oro AE. Eruptive xanthomas associated with olanzapine use. Arch Dermatol. 2003;139:1045-8.

10. Wirshing DA, Spellberg BJ, Erhart SM, Marder SR, Wirshing WC. Novel antipsychotics and new onset diabetes. Biol Psychiatry. 1998;44:77883. 